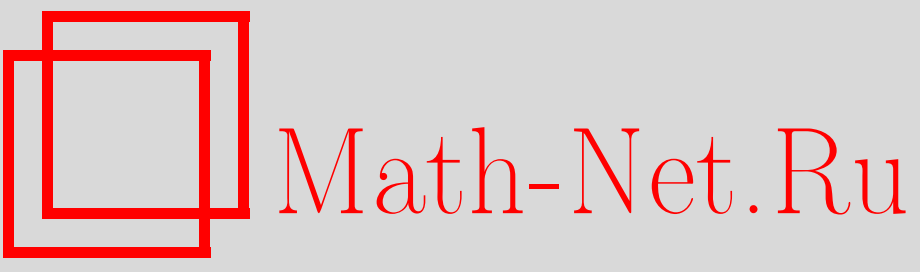

А. Р. Алимов, Связность солнц в пространстве $c_{0}$, Изв. РАН. Сер. матем., 2005, том 69, выпуск 4, 3-18

DOI: https://doi.org/10.4213/im645

Использование Общероссийского математического портала Math-Net.Ru подразумевает, что вы прочитали и согласны с пользовательским соглашением

http://www.mathnet.ru/rus/agreement

Параметры загрузки:

IP : 54.209 .52 .79

26 апреля 2023 г., 10:44:04 


\title{
А.Р. Алимов
}

\section{Связность солнц в пространстве $c_{0}$}

\begin{abstract}
Исследуется вопрос о связности солнца в пространстве $c_{0}$. Показано, что произвольное солнце $M$ в $c_{0} \mathrm{~m}$-связно (в смысле понятия, введенного А. Л. Брауном). Как следствие, получено, что $M$ монотонно линейно связно и что пересечение $M$ с произвольным шаром в $c_{0}$ монотонно линейно связно (в частности, линейно связно). С другой стороны, установлено, что каждое аппроксимативно компактное $\mathrm{m}$-связное множество является солнцем в $c_{0}$. Для $X=c_{0}, c, \ell^{\infty}$ показано, что пересечение солнца в $X$ с конечномерным координатным подпространством $H_{n} \subset X$ является $P$-ацикличным солнцем в $H_{n}$.

Библиография: 24 наименования.
\end{abstract}

\section{§1. Введение}

Мы изучаем геометрическое строение солнщ в бесконечномерных пространствах $c_{0}, c$ и $\ell^{\infty}$. Подобные вопросы рассматривались в [1]-[7]. Настояшая работа связана с работами [8] и [9], где, в частности, охарактеризованы чебьшевские множества в $\ell^{\infty}(n)$ и аппроксимативно компактные чебьшевские множества в $c_{0}$.

Для подмножества $M \neq \varnothing$ линейного нормированного пространства $X$ обозначим через $\rho(x, M)=\inf _{z \in M}\|x-z\|$ расстояние от $x$ до $M$ и через $P_{M} x=$ $\{y \in M \mid\|x-y\|=\rho(x, M)\}$ множество всех ближайших точек из $M$ для $x$.

Множество $M \subset X$ называется солнцем, если для любой точки $x \in X \backslash M$ сушествует точка $y \in P_{M} x$ (назьваемая солнечной) такая, что $y \in P_{M}[(1-\lambda) y+\lambda x]$ для всех $\lambda \geqslant 0$. Понятие солнца было введено в работах Н. В. Ефимова и С. Б. Стечкина (см., например, [10], [11]) и оказалось важным, в частности, для исследования чебышевских множеств. (О солнщах и чебышевских множествах см. работы [11]-[13], [1] и цитированную в них литературу.)

Далее $X$ - действительное линейное нормированное пространство.

Хорошо известно, что в гладком линейном нормированном пространстве всякое солнце выпукло [11, теорема 3.9$]$, поэтому вопрос о связности солнц представляет интерес только для негладких пространств. Первые результаты о связности солнщ были получены Л.П. Власовым и В.А. Кощеевым (см. §4, [11] и [13]). В настоящей работе мы изучаем геометрическую структуру солнщ в пространствах $X=c_{0}, c, \ell^{\infty}$.

В дальнейшем основным будет являться понятие т-связного подмножества банахова пространства $X$. В случае $\operatorname{dim} X<\infty \mathrm{m}$-связные подмножества $M$ пространства $X$ изучались А. Л. Брауном в [1], а в случае $\operatorname{dim} X=\infty-$ К. Франчетти и С. Роверси $[7]$ при дополнительном предположении ограниченной компактности множества $M$.

Напомним необходимые определения. Для $M \subset X$ через $\mathrm{m}(M)$ обозначим оболочку Банаха-Мазура множества $M$, т. е. пересечение всех замкнутых шаров 
$B(x, r)$ в $X$, содержаших $M$. Тогда $\mathrm{m}(M)$ - замкнутое множество, $M \subset \mathrm{m}(M)$. Подмножество $M \subset X$ называется $\mathrm{m}$-связны.м подмножеством $X$, если $\mathrm{m}(\{x, y\}) \cap$ $M \neq\{x, y\}$ для любых различных точек $x, y \in M$.

Браун [1], [2] показал, что m-связное замкнутое подмножество $M$ конечномерного пространства $X$ всегда линейно связно, $k$-связно для всех $k \in \mathbb{Z}_{+}, V$-ациклично (т. е. его пересечение с любым замкнутым шаром $B(x, r)$ в $X$ ациклично ${ }^{1}$ ) и $P$ - ациклично (т.е. для любой точки $x \in X$ множество $P_{M} x$ ее ближайших точек из $M$ ациклично); см. теорему А ниже. В теоремах 1 и 2 мы обобщаем это утверждение на $X=c_{0}, c, \ell^{\infty}$.

Для формулировки основных результатов введем необходимые определения. Пусть $X=c_{0}, c$ или $\ell^{\infty},\left(e_{i}\right)$ - стандартньй базис в $X$. Следуя [8] и [9], множество $H \subset X$ вида

$$
\operatorname{lin}\left\{e_{i} \mid i \in I\right\}, \quad I \subset \mathbb{N} \text { фиксировано, }
$$

будем назвать координатным подпространством пространства $X$. Аффинное координатное подпространство $H \subset X$ определяется как сдвиг координатного подпространства на вектор $x \in X$. Если $I$ в (1) конечно, то $n=\operatorname{dim} H=\operatorname{card} I<\infty$; множество всех $n$-мерных аффинных координатных подпространств $H \quad(n<\infty)$ будем обозначать $\mathrm{cAff} n(X)$.

Рассматривая аффинное координатное подпространство в $X=c_{0}, c$ или $\ell^{\infty}$, мы его всегда снабжаем нормой, индуцированной $\ell^{\infty}$-нормой на $X$.

Подмножество $M$ пространства $X$ функций, заданных на множестве $Q$, назовем монотонно линейно связным, если $M$ линейно связно и для любых точек $x$ и $y$ из $M$ найдется непрерывная кривая $k(t) \subset M$, соединяюшая их, координатные функции $k_{q}(t)$ которой монотонны по $t$ для любого $q \in Q$. K примеру, окружность линейно связна, но не монотонно линейно связна, а координатный крест $\left\{(x, y) \in \mathbb{R}^{2} \mid x \cdot y=0\right\}$ в $\mathbb{R}^{2}$ монотонно линейно связан. Несложно показать, что пересечение монотонно линейно связного подмножества функционального пространства, снабженного равномерной нормой, с произвольным шаром в этом пространстве монотонно линейно связно.

Обозначим $B(x, r)$ и $\stackrel{\circ}{B}(x, r)$ соответственно замкнутый и открытый шары с центром $x$ и радиусом $r$.

В теореме 1 показано, что если $M \subset c_{0}$ замкнуто и $\mathrm{m}$-связно, то множество $M$ и множества $M \cap B(x, r), M \cap \stackrel{B}{B}(x, r)$ и $P_{M} x$ монотонно линейно связны для любого выбора $x \in X$ и $r>0$.

Отметим, что монотонно линейно связное множество является m-связным в $X=c_{0}, c$ или $\ell^{\infty}$. Это будет следовать из предложения 1 .

В теореме 2 установлено, что пересечение $\mathrm{m}$-связного замкнутого подмножества пространства $X=c_{0}, c$ или $\ell^{\infty}$ с $n$-мерным координатным аффинным подпространством $H_{n}$ в $X, n \in \mathbb{N}$, является солнщем в $H_{n}$.

Изучая геометрические свойства солнц в конечномерных банаховых пространствах, Х. Беренс и Л. Хетцельт в работе [5] и А. Браун в [1] установили для

\footnotetext{
${ }^{1}$ Напомним, что множество $M$ называется (топологически) $k$-связным $\left(k \in \mathbb{Z}_{+}\right)$, если оно гомотопически тривиально над $k$-мерной сферой; множество $M$ ациклично, если оно непусто, компактно и его группы гомологий $H_{q}, q \geqslant 1$, и приведенная группа $\widetilde{H}_{0}$ тривиальны. Напомним, что стягиваемое (в частности, выпуклое) компактное подмножество линейного нормированного пространства ациклично; ацикличное множество связно; гомеоморфиный образ ацикличного множества ацикличен.
} 
замкнутого непустого множества $M \subset \mathbb{R}^{n}$, что $^{2}$ :

$$
\begin{aligned}
M-\text { солнце в } \ell^{\infty}(n) & \Leftrightarrow M \ell^{1} \text {-выпукло } \Leftrightarrow M \mathrm{~m} \text {-связно в } \ell^{\infty}(n) \\
& \Leftrightarrow M \text { монотонно линейно связно. }
\end{aligned}
$$

Ниже (предложение 3 ) мы покажем, что существует норма $|\cdot|$ на $X=c_{0}, c, \ell^{\infty}$ такая, что для данных $z, x, y \in X$ выполнено

$$
z \in \mathrm{m}(x, y) \Leftrightarrow z \text { находится }|\cdot| \text {-между } x \text { и } y,
$$

где последнее означает, что $|x-y|=|x-z|+|z-y|$.

Используя (3), мы обобщаем (2) на случай произвольных солнц в $c_{0}$ (теоремы 1 , $3,5)$ и частью для случая $X=c, \ell^{\infty}$ (теоремы 2, и 6 ).

Напомним, что подмножество $M \subset X$ называется аппроксимативно компактнылм [10], [11], если для любой точки $x \in X$ и для любой последовательности $\left(y_{n}\right)$ из $M$ такой, что $\left\|x-y_{n}\right\| \rightarrow \rho(x, M)$, найдется подпоследовательность, сходяшаяся к элементу из $M$. Отметим, что для такого $M$ множество $P_{M} x$ всегда непусто и компактно (см. [11], [14]).

В теореме 3 показано, что произвольное солнце в $c_{0}$ m-связно; обратно, m-связное аппроксимативно компактное непустое подмножество пространства $c_{0}$ является солнцем. Как следствие, солнще в $c_{0}$ монотонно линейно связно (теорема 5).

Таким образом, для аппроксимативно компактного множества в $c_{0}$ его солнечность эквивалентна его т-связности (монотонной линейной связности).

Напомним, что множество $M \subset X$ называется чебышевским, если любая точка из $X$ имеет только одну ближайшую точку $P_{M} x$ из $M$.

В теореме 4 установлено, что $\mathrm{m}-$ связное чебышевское множество в $c_{0}$ является солнцем.

В теореме 6 изучен вопрос о солнечности пересечения солнца $M$ в пространствах $c_{0}, c$ или $\ell^{\infty}$ с конечномерными координатными подпространствами $H_{n}$. В частности, если $H \cap H_{n} \neq \varnothing$, то $M \cap H_{n}-$ m-связное солнце в $H_{n}$.

\section{§ 2. Вспомогательные утверждения}

Помимо m-связных множеств ${ }^{3}$ в работе будет использоваться другое важное понятие, также введенное А. Брауном [1]. Линейное нормированное пространство $X$ называется $(B M)$-пространством, если

$$
B(0,\|x\|) \cap(\mathrm{m}(x, y) \backslash\{x\}) \neq \varnothing, \quad \text { когда }[x, x-y] \cap \stackrel{\circ}{B}(0,\|x\|)=\varnothing .
$$

Класс $(B M)$-пространств содержит в себе все гладкие пространства, все двумерные пространства с полигональным единичным шаром, пространство $\ell^{\infty}(n)$

\footnotetext{
${ }^{2}$ Подмножество $M \subset \mathbb{R}^{n}$ называется $\ell^{1}$-выпуклымм, если для любых различных $x, y \in M$ существует точка $z \in M, z \neq x, y$, такая, что $|x-y|_{1}=|x-z|_{1}+|z-y|_{1} ;$ здесь $\left.|\cdot|\right|_{1}-$ обычная $\ell^{1}$-норма на $\mathbb{R}^{n}$.

3 Буква "m" в названии отмечает вклад Мазура в основание теории. Он исследовал пространства, в которых выполнено $\mathrm{m}(M)=M$ для каждого замкнутого ограниченного выпуклого множества $M$; такие пространства называются пространствами со свойством пересечения Мазура (см. [15], [16]). Мазур установил это свойство для евклидовых пространств.
} 
и замкнут по отношению к формированию конечной $\ell^{\infty}$-прямой суммы $[1, \S 5]$ и бесконечной $c_{0}$-прямой суммы сепарабельных $(B M)$-пространств [7, теорема 8.7]. Далее, в предложении 2 , мы установим, что пространства $c_{0}, c$ и $\ell^{\infty}$ также являются $(B M)$-пространствами.

Используя это понятие, Браун доказал, что солнща в конечномерных $(B M)$-пространствах удовлетворяют сильным когомологическим условиям связности (являясь $c l c^{\infty}$-когомологически локально связными и, в частности, $P$-ацикличными и $R_{\delta}$-множествами по Ароншайну).

Следующее предложение было доказано в [5] для $X=\ell^{\infty}(n)$ и в $[1$, теорема 3.1$]$ для произвольных конечномерных пространств.

ПрЕДЛОЖЕНИЕ 1. Пусть $X=c_{0}$, с или $\ell^{\infty}, x, y \in X$. Тољда

$$
\mathrm{m}(x, y)=Z(x, y), \quad Z(x, y)=\left\{z \in X \mid z^{(i)} \in\left[x^{(i)}, y^{(i)}\right], i \in \mathbb{N}\right\}
$$

Прежде чем доказывать предложение 1 , напомним определение опорного конуca $\stackrel{\circ}{K}(y, x)$ к шару $B(x,\|x-y\|)$ в его граничной точке $y$ (см. [11, гл. 3$])$ :

$$
\stackrel{\circ}{K}(y, x)=\bigcup_{r>0} \stackrel{\circ}{B}(-r y+(r+1) x,(r+1)\|x-y\|) .
$$

Будем также использовать следующее эквивалентное представление для конуса $\stackrel{\circ}{K}(y, x)$. Рассмотрим точку $p$ на единичной сфере $S$. Через $\Sigma_{p}$ обозначим множество всех функционалов из $S^{*}$, достигающих максимум на $S$ в $p$ (где $S^{*}$ - единичная сфера сопряженного пространства). Понятно, что $\Sigma_{p}$ непусто и является вьпуклым $w^{*}$-компактным множеством; по теореме Крейна-Мильмана множество $\mathcal{E}_{p}$ его экстремальных точек непусто. Далее, для различных точек $x, y \in X$ определим точку $p=(y-x) /\|y-x\|$. Тогда [11, лемма 3.1] имеем

$$
\begin{aligned}
\stackrel{\circ}{K}(y, x) & =\left\{z \in X \mid f(z)<f(y) \forall f \in \Sigma_{p}\right\} \\
& =\left\{z \in X \mid f(z)<f(y) \forall f \in \mathcal{E}_{p}\right\} .
\end{aligned}
$$

Важное свойство солнц (точка, не принадлежащая солнцу, отделяется от него посредством выпуклого открытого конуса) дается в следующем утверждении (см., например, [11, гл. 3]); мы воспользуемся им позже.

Лемма А. Подмножсество $M \subset X$ является солнцем в $X$ тогда и только тогда, когда для любой точки $x \notin M$ существует точка $y \in P_{M} x$ такая, что $\stackrel{\circ}{K}(y, x) \cap M=\varnothing$.

ДоКАЗАТЕЛЬСТВо ПРЕДЛОЖЕНИЯ 1 . Пусть $z \in Z(x, y)$. Рассмотрим шар $B(v, r) \ni x, y$. Понятно, что $\|v-z\| \leqslant \max \{\|x-z\|,\|y-z\|\}$, откуда $z \in B(v, r)$, и, следовательно, $z \in \mathrm{m}(x, y)$.

Пусть теперь $z \notin Z(x, y)$. Тогда сушествует номер $i$ такой, что $z^{(i)} \notin\left[x^{(i)}, y^{(i)}\right]$. Без ограничения общности считаем, что $z^{(i)}>\max \left\{x^{(i)}, y^{(i)}\right\}+a, a>0$. На единичной сффере $S$ рассмотрим точку $s=e_{i}$. Хорошо известно, что $s$-точка гладкости для $S$ (в $\left[17\right.$, с. 352] показано, что точками гладкости сферы $S$ для $X=\ell^{\infty}$ являются в точности точки $\sigma e_{k}+x^{\prime}$, где $|\sigma|=1$ и $\left.\left\|x^{\prime}\right\|<1\right)$. По определению опорная гиперплоскость $H_{s}$ к $S$ в точке $s$ единственна, поэтому опорный конус $\stackrel{\circ}{K}(s, 0)$ 
к $S$ в точке $s$ является открытым полупространством с границей $H_{s}$. В силу (6) имеем

$$
\stackrel{\circ}{K}(s, 0)=\left\{u \in X \mid u^{(i)}<1\right\} .
$$

Определим точку $\bar{z}=\left(\bar{z}^{(j)}\right)$, где $\bar{z}^{(j)}=z^{(j)}$ для $j \neq i$ и $\bar{z}^{(i)}=z^{(i)}-a / 2$, и сдвинем $\stackrel{\circ}{K}(s, 0)$ и $H_{s}$ на вектор $\bar{z}-s$; тогда

$$
\stackrel{\circ}{K}(\bar{z}, \bar{z}-s)=\left\{u \mid u^{(i)}<z^{(i)}-\frac{a}{2}\right\}
$$

будет опорным конусом к шару $B(\bar{z}-s, 1)$, а $H_{s}=\bar{z}-s-$ его границей. Вследствие $(5)$ конус $\stackrel{\circ}{K}(\bar{z}, \bar{z}-s)$ представляется в виде объединения открытых шаров. Поскольку $x, y \in \stackrel{\circ}{K}(\bar{z}, \bar{z}-s)$, то каждый шар из (5) содержит $x$ и $y$ для всех достаточно больших $r$. Следовательно, $z \notin \mathrm{m}(x, y)$, что и требуется доказать.

Используя те же аргументы, можно установить аналог предложения 1 для пространств $C(Q)$ и $C_{0}(Q)$.

Следующий результат доказан в [1] для $X=\ell^{\infty}(n)$.

ПРЕДЛОЖЕНИЕ 2. Пространства $c_{0}, c, \ell^{\infty}$ являются (BM)-пространствами.

ДокаЗАТЕЛЬство. Пусть $X=c_{0}, c$ или $\ell^{\infty}$. Рассмотрим $x \in X,\|x\|=1$, и зафиксируем произвольно точку $y \in X$ такую, что

$$
[x, x-y] \cap \stackrel{\circ}{B}(0,\|x\|)=\varnothing .
$$

Проверим, что выполнено (4).

Для простоты считаем, что $x^{(i)} \geqslant 0$ для всех $i$. Докажем, что найдется номер $j$ такой, что $y^{(j)}<1$ (без этого предположения нам следовало бы установить существование такого $i$, что $y^{(i)}<1$ в случае $x^{(i)} \geqslant 0$ или $y^{(i)}>-1$ в случае $\left.x^{(i)}<0\right)$.

Предположим противное: пусть $y^{(i)} \geqslant 1$ для любого $i \in \mathbb{N}$. Рассмотрим точку $x_{\alpha}=x-\alpha y \in(x, x-y), 0<\alpha<1$. Тогда

$$
x_{\alpha}^{(i)} \leqslant 1-\alpha, \quad x_{\alpha}^{(i)} \geqslant x^{(i)}-\alpha\|y\| \geqslant-\alpha\|y\| .
$$

Если $\alpha \rightarrow 0$, то найдутся $\alpha_{0}>0$ и $0<\delta<1$ такие, что $-\alpha_{0}\|y\|>-1+\delta$. Поэтому

$$
\left\|x_{\alpha_{0}}^{(i)}\right\| \leqslant \min \left\{1-\delta, 1-\alpha_{0}\right\}
$$

откуда $\left\|x_{\alpha_{0}}\right\|<1$. Однако $x_{\alpha_{0}} \in(x, x-y)$ - противоречие с (7).

По доказанному найдется такой номер $j$, что $y^{(j)}<1$. Определим точку $u$ такую, что $u^{(i)}=x^{(i)}$ для $i \neq j$ и $u^{(j)}=y^{(j)}$. Ясно, что $u \in X$. По предложению 1 имеем $u \in \mathrm{m}(x, y)$ и $[u, x] \subset \mathrm{m}(x, y)$. Пусть $u_{\beta}=\beta x+(1-\beta) u, 0<\beta<1$. Тогда

$$
-(1-\beta)\|y\| \leqslant(1-\beta) y^{(j)} \leqslant u_{\beta}^{(j)}<\beta x^{(j)}+(1-\beta) \leqslant 1 .
$$

Отсюда вытекает, что $u_{\beta} \in B(0,1)$ для всех достаточно больших $\beta \rightarrow 1$. Следовательно, $u_{\beta} \in(\mathrm{m}(x, y) \backslash\{x\}) \cap B$, что и требуется доказать.

Рассмотрим метрическое пространство $(X, d)$, или, в частном случае, линейное нормированное пространство $(X,|\cdot|)$. Напомним [18], [1], что точка $z \in X$ расположена $d$-между (или $|\cdot|$-между) точками $x, y \in X$, если $d(x, y)=d(x, z)+$ $d(y, z)$. Множество $M \subset X$ называется $d$-выпукльлм (или $|\cdot|$-вылуккльлм), если для любых $x, y \in M$ множество $M \backslash\{x, y\}$ содержит точки, расположенные $d$-между $(|\cdot|$-между) точками $x$ и $y$. 
ПРЕДЛОЖЕНИЕ 3. СУществует норма $|\cdot|$ на $X=c_{0}, c, \ell^{\infty}$ такая, что для всех $x, y, z \in X$

$$
z \in \mathrm{m}(x, y) \Leftrightarrow z \text { находится }|\cdot| \text {-междуу } x \text { и } y .
$$

Такую норму будем называть ассоциированной (см. [1]). Отметим, что утверждения, аналогичные (8), возникают в римановой геометрии [19].

Ниже, если не оговорено противное, через $\|\cdot\|$ будем обозначать $\ell^{\infty}$-норму на пространстве последовательностей.

ДОКАЗАТЕЛЬСТВО ПРЕДЛОЖЕНИЯ 3. БУДем испольЗовать фрагменТЫ схем из [1]. Пусть $\alpha_{i}>0$ для всех $i=1,2, \ldots$ и $\alpha_{1}+\alpha_{2}+\ldots<\infty$. Определим на $X$ следующую норму:

$$
|x|=\sum_{i=1}^{\infty} \alpha_{i}\left|x^{(i)}\right| .
$$

Ясно, что $|x| \leqslant\|x\|\left(\alpha_{1}+\alpha_{2}+\ldots\right)$. Тогда условия

(i) $z \in \mathrm{m}(x, y)$,

(ii) $z^{(i)} \in\left[x^{(i)}, y^{(i)}\right]$ для всех $i \in \mathbb{N}$,

(iii) $\left|x^{(i)}-y^{(i)}\right|=\left|x^{(i)}-z^{(i)}\right|+\left|z^{(i)}-y^{(i)}\right|$ для всех $i \in \mathbb{N}$,

(iv) $|x-y|=|x-z|+|z-y|$ эквивалентны по предложению 1.

Для пространства $X=\ell^{\infty}(n)$ ясно, что $\ell^{1}$-норма является ассоциированной к равномерной норме $\|\cdot\|$. Из предложения 3 , рассуждая аналогично [1], имеем, что если $|\cdot|$ - ассоциированная норма (9) на $X=c_{0}, c$ или $\ell^{\infty}$, то в силу (8) выполнено следующее утверждение:

$(*)$ замкнутое подмножество пространства $X$ является m-связным, если и только если оно $|\cdot|$-вьпукло.

ПРЕДЛОЖЕНИЕ 4. 1) Каждое из пространств $c_{0}$, с и $\ell^{\infty}$ не является полньлм по отношению $к$ ассоциированной норме $|\cdot|$.

2) Для любых $u, v \in c_{0}$ или $u, v \in \ell^{\infty}$ множество $\mathrm{m}(u, v)$ является полным пространством по отношению $\kappa|\cdot|$.

ДОКАЗАТЕЛЬСТво. 1) Хорошо известно, что для любой последовательности $\left(\alpha_{i}\right)$ положительных чисел такой, что $\sum \alpha_{j}<\infty$, существует последовательность $\left(d_{n}\right), d_{n}>0, d_{n} \uparrow+\infty$, такая, что $\sum \alpha_{j} d_{j}<\infty$. Определим $x_{n}=$ $\left(d_{1}, \ldots, d_{n}, 0, \ldots\right)$. Тогда $\left(x_{n}\right)$ - последовательность Коши в норме $|\cdot|$, которая не может сходиться к элементу из $\ell^{\infty}$.

2) Пусть $\left(x_{n}\right)$ - последовательность Коши в $(\mathrm{m}(u, v),|\cdot|)$. Рассмотрим ее предельную точку $x \in\left\{z=\left(z^{(i)}\right)\left|\sum \alpha_{i}\right| z^{(i)} \mid<\infty\right\}$ в $|\cdot|$-пополнении множества $m(u, v)$. Предположим, что $x \notin \mathrm{m}(u, v)$. Тогда по предложению 1 существует номер $j$ такой, что выполнен случай а) $x^{(j)}>\max \left\{u^{(j)}, v^{(j)}\right\}+\gamma$ или случай б) $x^{(j)}<\min \left\{u^{(j)}, v^{(j)}\right\}-\gamma, \gamma>0$.

Рассмотрим случай а) и выберем $\varepsilon>0$ такое, что $\gamma-\varepsilon / \alpha_{j}>0$. Для такого $\varepsilon$ выберем номер $N$ такой, что $\left|x_{n}-x\right|<\varepsilon$ при всех $n>N$. Отсюда $\alpha_{j}\left|x_{n}^{(j)}-x^{(j)}\right|<\varepsilon$. Следовательно,

$$
x_{n}^{(j)}>x^{(j)}-\frac{\varepsilon}{\alpha_{j}}>\max \left\{u^{(j)}, v^{(j)}\right\}-\frac{\varepsilon}{\alpha_{j}}+\gamma>\max \left\{u^{(j)}, v^{(j)}\right\},
$$


что невозможно, поскольку $x_{n} \in \mathrm{m}(u, v)$, поэтому предположение, что $x \notin \mathrm{m}(u, v)$, было неверно.

Случай б) рассматривается аналогично.

ПРЕДЛОЖЕНИЕ 5 (см. также [7]). Пусть $u, v \in c_{0}$. Тогда:

1) $\mathrm{m}(u, v)$ - компактное подмножсество пространства $c_{0}$;

2) если $M \subset c_{0}$ замкнуто, то $\mathrm{m}(u, v) \cap M$ является $|\cdot|$-компактным множеством.

ДокАЗАТЕЛЬСтво. 1) Утверждение следует из предложения 1 и критерия компактности в $c_{0}$.

2) Рассмотрим произвольную последовательность $\left(x_{n}\right) \subset M \cap \mathrm{m}(u, v)$. Пусть $x$ - ее $\|\cdot\|$-предельная точка в $\mathrm{m}(u, v)$, существующая в силу 1$)$. Поскольку $M$ замкнуто, то $x \in M \cap \mathrm{m}(u, v)$. Теперь $|\cdot|$-компактность $M$ следует из неравенства $|y| \leqslant\|y\| \sum \alpha_{i}$, которое выполнено для любого $y \in c_{0}$.

\section{§ 3. Свойства m-связных множеств}

Важным результатом при исследовании m-связных множеств является

ЛЕмма В ([18], см. также [1, с. 93]). Пусть $(Y, d)-d$-вьлууклое компактное метрическое пространство. Тогда для $x$ и у в $Y$ существует изометрия $f:[0, d(x, y)] \rightarrow Y$ такая, что $f(0)=x$ и $f(d(x, y))=y$. Как следствие, $Y$ линейно связно.

Следуюший результат доказан в [5] для $\ell^{\infty}(n)$. Напомним, что в силу $(*)$ замкнутое множество $M \subset c_{0}$ является m-связным тогда и только тогда, когда $M$ $|\cdot|$-выпукло, где норма $|\cdot|$ определена в $(9)$.

ПРЕДЛОЖЕНИЕ 6. Для любъх точек $x, y$ из т-связного замкнутого множества $M \subset c_{0}$ существует непрерывная кривая $k(t) \subset M, 0 \leqslant t \leqslant|x-y|$, связывающая $x$ и функции $k^{(i)}(t)$ кривой $k(t)$ монотонны по $t$ для каждого $i \in \mathbb{N}$.

Такую кривую $k(t)$ назовем монотонной дугой.

В введенных обозначениях из предложения 6 следует, что т-связное замкнутое подмножество в $c_{0}$ является монотонно линейно связным. Таким образом, для замкнутого множества $M \subset c_{0}$ имеем

\section{$M \mathrm{~m}$-связно $\Leftrightarrow M$ монотонно линейно связно.}

ДОКАЗАТЕЛЬСТвО ПРЕДЛОЖЕНИЯ 6. Рассмотрим $x, y \in M$. По предположению множество $M \subset X$ является $\|\cdot\|$-замкнутым, поэтому $M \cap \mathrm{m}(x, y)$ замкнуто. Из предложения 4 множество $\mathrm{m}(x, y)|\cdot|$-полно. По предложению 5 множество $M \cap \mathrm{m}(x, y)|\cdot|$-замкнуто, поэтому мы можем применить лемму В к множеству $M \cap \mathrm{m}(x, y)$, получая монотонную дугу $k(\cdot)$, соединяющую $x$ и $y$.

Докажем монотонность по $t$ координатных функций $k^{(i)}(t)$. Без ограничения обшности считаем, что $x=0, y^{(i)} \geqslant 0$ для любого $i,|y|=1$. Для любых $\beta_{1}, \beta_{2} \in(0,1), \beta_{1} \leqslant \beta_{2}$, по лемме В сушествуют точки $x_{\beta_{1}}, x_{\beta_{2}} \in k(\cdot)$ такие, что 
$\left|x_{\beta_{1}}\right|=\beta_{1},\left|y-x_{\beta_{1}}\right|=1-\beta_{1},\left|x_{\beta_{2}}\right|=\beta_{2},\left|y-x_{\beta_{2}}\right|=1-\beta_{2}$. По предложению 1 имеем $0 \leqslant x_{\beta_{1}}^{(i)}, x_{\beta_{2}}^{(i)} \leqslant y^{(i)}$. Следовательно,

$$
\begin{aligned}
|y| & =\sum \alpha_{i} y^{(i)}=\left|x_{\beta_{1}}\right|+\left|x_{\beta_{1}}-x_{\beta_{2}}\right|+\left|y-x_{\beta_{2}}\right| \\
& =\sum \alpha_{i}\left(x_{\beta_{1}}^{(i)}+\left|x_{\beta_{1}}^{(i)}-x_{\beta_{2}}^{(i)}\right|+y^{(i)}-x_{\beta_{2}}^{(i)}\right),
\end{aligned}
$$

откуда $x_{\beta_{1}}^{(i)} \leqslant x_{\beta_{2}}^{(i)}$ для всех $i$, что и требуется доказать.

ЗАмечАние 1 . В пространстве $C[0,1] \mathrm{m}$-связное множество может быть несвязным. Соответствующий пример построен в [7, с. 12]. Пусть $M=M_{1} \cup M_{-1}$, где $M_{\sigma}=\{x \in C[0,1] \mid x(0)=\sigma\}, \sigma= \pm 1$. Тогда $M$ состоит из двух выпуклых компонент и поэтому несвязно. Однако множество $M \mathrm{~m}$-связно. Отметим, что $M$ не является солнщем. Аналогичный пример можно построить в $X=c$.

Teоpema A [1], [2]. Пусть X - конечномерное линейное нормированное пространство, и пусть $\varnothing \neq M \subset X$ замкнуто. Каждое из четырех условий:

1) $M \mathrm{~m}$-связно,

2) $M \cap B(x, r) k$-связно для всех $k=0,1,2, \ldots$ и любых $x \in X, r>0$,

3) $M V$-ациклично,

4) $М$ Р-ациклично, влечет следующее:

5) $M-$ солние.

Если $X$ имеет размерность два или является (ВМ)-пространством, то все условия эквивалентны.

Отметим, что существует конечномерное негладкое пространство $X$, в котором всякое $\mathrm{m}$-связное замкнутое множество выпукло. Поэтому в этом пространстве существует не т-связное солнще (см. [1], [3]). В [3, теорема 1.2] охарактеризованы конечномерные полиэдральные пространства, в которых каждое солнце m-связно.

$\mathrm{B}$ теоремах 1 и 2 мы частично обобшаем теорему $\mathrm{A}$ на случай пространств $c_{0}, c$ и $\ell^{\infty}$. Утверждение теоремы 1 вытекает из предложений 1 и 6.

Teорема 1. Пусть $M \subset c_{0}$ замкнуто и $\mathrm{m}$-связно. Тогда множество $M$ и множества $M \cap B(x, r), M \cap \stackrel{B}{(x, r)}$ и $P_{M} x$ монотонно линейно связны для любого выбора $x \in X$ и $r>0$.

Tеорема 2. Пусть $M-\mathrm{m}$-связное замкнутое непустое подмножество пространства $X=c_{0}$, с или $\ell^{\infty}$. Eсли $H_{n} \in \operatorname{cAff}_{n}(X)$ - n-мерное координатное аффинное подпространство в $X, n \in \mathbb{N}, B_{H_{n}}(x, r)$ - замкнутый шар в $H_{n}$ u $B_{H_{n}}(x, r) \cap M \neq \varnothing$, mo:

1) $M \cap B_{H_{n}}(x, r)$ ациклично;

2) $M \cap H_{n}-$ солние в $H_{n}$.

Кроме того, $M \cap H_{n}$ монотонно линейно связно.

ДоКАЗАТЕЛЬСТво. Поскольку $\operatorname{dim} H_{n}=n<\infty$, то отождествим $H_{n}$ с $\ell^{\infty}(n)$. Выберем $u, v \in H_{n}$. По предложению 1 имеем $\mathrm{m}(u, v) \subset H_{n}$. Следовательно, $M \cap H_{n}$ также m-связно. Теперь монотонная линейная связность $M \cap H_{n}$ следует из предложения 6. 
Зафиксируем замкнутьй шар $B_{H_{n}}(x, r)$ в $H_{n}$. Снова применяя предложение 1 , получим, что $M \cap B_{H_{n}}(x, r)$ m-связно. В [1, с. 93] установлено, что непустое ограниченное замкнутое $\mathrm{m}$-связное подмножество конечномерного линейного нормированного пространства $Y$ ациклично. Полагая $Y=H_{n}$, получаем ацикличность множества $M \cap B_{H_{n}}(x, r)$.

Солнечность множества $M \cap H_{n}$ в $H_{n}$ следует из [11, теорема 4.4], где показано, что ограниченно-компактное $P$-ацикличное подмножество банахова пространства является солнщем. Теорема 2 доказана.

\section{§4. Свойства солнц в пространствах $c_{0}, c$ и $\ell^{\infty}$}

Напомним, что известно о связности солнц в линейных нормированных пространствах. Этот вопрос изучался многими авторами (см., например, [13]). Л.П. Власов [11, теорема 3.9] показал, что солнце в $X$ вьпукло, если и только если единичный шар в $X$ является гладким. Поэтому вопрос о связности солнц представляет интерес только в негладких пространствах.

В. А. Кощеев [21] установил, что солнще в конечномерном банаховом пространстве $X_{n}$ связно. Браун [1], [2], развивая рассуждения Кошеева, показал, что солнце $M$ в $X_{n}$ линейно связно и, следовательно, локально линейно связно. Более того [2, теорема 3], сушествуют положительные константы $C$ и $\alpha$, зависящие от пространства $X_{n}$, такие, что для любых $x_{0}, y_{0} \in M$ сушествует путь $s:[0,1] \rightarrow M$, связывающий $x_{0}$ с $y_{0}$, такой, что

$$
\|s(\xi)-s(\eta)\| \leqslant C\left\|x_{0}-y_{0}\right\| \cdot|\xi-\eta|^{\alpha}
$$

для всех $\xi, \eta \in[0,1]$.

В бесконечномерном случае Кошеев [22] установил, что компактное солнце в банаховом пространстве связно и что произвольное солнце в равномерно неквадратном $^{4}$ банаховом пространстве связно [22, следствие 1]. В работе [23] им построен пример несвязного солнца в некотором банаховом пространстве.

Вопрос о том, является ли произвольное солнце в конечномерном пространстве размерности $\geqslant 3$ стягиваемым, $P$-ацикличным или, по крайней мере, $V$-связным, является открытым (см. [2], [3], [13]). Однако в некоторых конечномерных пространствах $X$ ответ на этот вопрос положителен. По теореме А в конечномерном $(B M)$-пространстве $X$ солнца оказываются m-связными и поэтому $P$-ацикличными. Аналогичный результат верен для произвольных солнщ в $X=c_{0}$.

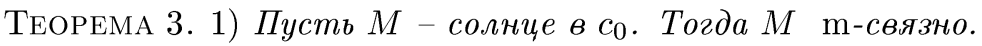

2) т-связное аппроксимативно компактное непустое подмножсество пространства с является солнием.

В [7, теорема 7.4] утверждения теоремы 3 установлены для ограниченно-компактных множеств $M$ (т. е. для множеств $M$ со свойством: $M \cap B(x, r)$ компактно для любого выбора $x$ и $r>0)$; понятно, что каждое ограниченно-компактное множество является аппроксимативно компактным, но не наоборот.

\footnotetext{
${ }^{4}$ Напомним, что линейное нормированное пространство $(X,\|\cdot\|)$ равномерно неквадратно, если найдется $\varepsilon>0$ такое, что $\|(x+y) / 2\| \leqslant 1-\varepsilon$ или $\|(y-x) / 2\| \leqslant 1-\varepsilon$ для всех $x, y \in X,\|x\|=\|y\|=1$. Понятно, что $c_{0}, c$ и $\ell^{\infty}$ не являются такими пространствами.
} 
ЗАмЕчАнИЕ 2. Легко построить замкнутое выпуклое (и потому $-\mathrm{m}$-связное) множество в $c_{0}$, не являюшееся солнцем (достаточно взять гиперплоскость, определяемую функционалом, не достигающим своей нормы). Вопрос, будет ли произвольное т-связное множество существования солнцем в $c_{0}$, является открытым.

ДОКАЗАТЕЛЬСТво ТЕОРЕмЫ 3. Для доказательства утверждения 1) используются некоторые схемы из [1]. Предположим противное и рассмотрим солнце $M \subset c_{0}$, не являющееся m-связным. Тогда по определению существуют различные точки $u, v \in M$ такие, что

$$
(\mathrm{m}(u, v) \backslash\{u, v\}) \cap M=\varnothing .
$$

Рассмотрим определенную в (9) ассоциированную норму $|\cdot|$ на $c_{0}$.

1. Для каждого $\varepsilon>0$ положим

$$
\mathcal{O}_{\varepsilon}(M)=\left\{x \in X \mid \rho(x, M):=\inf _{z \in M}\|x-z\| \leqslant \varepsilon\right\}, \quad A_{\varepsilon}=\mathrm{m}(u, v) \cap \mathcal{O}_{\varepsilon}(M)
$$

Покажем, что для любого $\varepsilon>0$ существует $x_{\varepsilon} \in A_{\varepsilon}$ такое, что

$$
\left|x_{\varepsilon}-u\right|=\left|x_{\varepsilon}-v\right|=\frac{1}{2}|u-v| \text {. }
$$

Отсюда будет следовать, что если $\bar{x}-|\cdot|$-предельная точка для $x_{\varepsilon}$, существующая по предложению 5 , то $\bar{x} \in(\mathrm{m}(u, v) \backslash\{u, v\}) \cap M$, что противоречит предположению (10).

Мы можем считать, что $\varepsilon<\|u-v\| / 2$. Если $0<\theta<\varepsilon /\|u-v\|$, то $v+$ $\theta(u-v) \in A_{\varepsilon}$ и

$$
|(v+\theta(u-v))-u|>\frac{1}{2}|u-v| .
$$

Предположим, что $x_{0} \in A_{\varepsilon}, x_{0} \neq u, v,\left|x_{0}-u\right| \geqslant|u-v| / 2$ и что $y-$ солнечная точка для $x_{0}$. В силу (10) $x_{0} \notin M$, поэтому $y \neq x_{0}$. Для простоты обозначений считаем $x_{0}=0$.

2. Поскольку $y$ - солнечная точка для 0 и $u \in M$, то $[y, u] \cap \stackrel{\circ}{B}(0,\|y\|)=\varnothing$ (см. $[11$, лемма 3.1$])$; это эквивалентно тому, что $[y, y-(y-u)] \cap \stackrel{\circ}{B}(0,\|y\|)=\varnothing$. Так как по предложению $2 c_{0}$ является $(B M)$-пространством, то из (4) следует

$$
B(0,\|y\|) \cap(\mathrm{m}(y, y-u) \backslash\{y, y-u\}) \neq \varnothing
$$

что эквивалентно $B(y,\|y\|) \cap(\mathrm{m}(0, u) \backslash\{0, u\}) \neq \varnothing$. Предположим, что $w$ содержится во множестве $B(y,\|y\|) \cap(\mathrm{m}(0, u) \backslash\{0, u\})$. Тогда $[0, w]$ тоже содержится в нем. Кроме того, $\|w-y\| \leqslant\|y\| \leqslant \varepsilon$, так что $[0, w] \subset \mathcal{O}_{\varepsilon}(M)$, и $(0, w] \subset \mathrm{m}(0, u) \backslash$ $\{0, u\}$, откуда по предложению 3 имеем

$$
\left|w^{\prime}-u\right|<|u| \text { для любой точки } w^{\prime} \in(0, w] .
$$

3. Убедимся, что множество $Z=\{z \in \mathrm{m}(u, v)|| z-u|\geqslant(1 / 2)| u-v \mid\}|\cdot|$-замкнуто. Действительно, если $\left(x_{n}\right) \subset Z$, то в силу $|\cdot|$-компактности множества $\mathrm{m}(u, v)$ (предложение 5) имеем, что $\left|x_{n_{k}}-x\right| \rightarrow 0$ для некоторого $x \in \mathrm{m}(u, v)$. Не ограничивая общности считаем $\left|x_{n}-x\right| \rightarrow 0$. Предположим, что $x \notin Z$. Тогда 
$|x-u|+\gamma=(1 / 2)|u-v|$ при некотором $\gamma>0$. Для $0<\varepsilon<\gamma$ выберем номер $N$ такой, что $\left|x_{n}-x\right|<\varepsilon$ для всех $n \geqslant N$. Имеем

$\left|x_{n}-u\right| \leqslant\left|x_{n}-x\right|+|x-u|<\varepsilon+|x-u|<\gamma+|x-u|=\frac{1}{2}|u-v| \leqslant\left|x_{n}-u\right|, \quad n \geqslant N$.

Полученное противоречие показывает, что $Z$ замкнуто.

Рассмотрим

$$
a_{\varepsilon}=\inf \left\{|z-u| \mid z \in A_{\varepsilon} \text { и }|z-u| \geqslant \frac{1}{2}|u-v|\right\} .
$$

Поскольку $M$ непусто, то $\mathcal{O}_{\varepsilon}(M)$ тоже замкнуто (так как функция расстояния до непустого множества непрерывна), откуда множество $A_{\varepsilon}$ является $|\cdot|$-компактным по предложению 5 . По доказанному вьше $Z$ замкнуто, поэтому в (12) инфимум достигается, скажем, в точке $x_{\varepsilon}$. Отсюда $x_{\varepsilon} \in A_{\varepsilon}$ и $\left|x_{\varepsilon}-u\right| \geqslant(1 / 2)|u-v|$.

Далее,

$$
a_{\varepsilon}=\left|u-x_{\varepsilon}\right|=\frac{1}{2}|u-v|=\left|v-x_{\varepsilon}\right|,
$$

в противном случае из (11) получим точку $w^{\prime} \in \mathcal{O}_{\varepsilon}(M) \cap \mathrm{m}(u, v),\left|w^{\prime}-u\right| \geqslant$ $|u-v| / 2$, и окажется, что $w^{\prime}$ находится $|\cdot|$-ближе к $u$, чем $x_{\varepsilon},-$ противоречие с (12).

Докажем утверждение 2$)$. Пусть $0 \notin M, \rho(0, M)=1$. Поскольку $M$ аппроксимативно компактно, то $P_{M} 0 \neq \varnothing$ компактно; кроме того, множество

$$
I=\left\{i \in \mathbb{N} \mid \text { существует точка } y \in P_{M} 0 \text { такая, что }\left|y^{(i)}\right|=1\right\}
$$

непусто и конечно. Обозначим $k=\max \{i \mid i \in I\}$.

Докажем, что $M$ является солнцем в $c_{0}$, для этого воспользуемся конечномерным результатом (2) для $\ell^{\infty}(k)$. Определим

$$
\begin{aligned}
\widehat{M} & =M \cap\left\{z \mid-1<z^{(j)}<1 \quad \forall j \geqslant k+1\right\}, \\
M^{\prime} & =\operatorname{pr}_{\mathbb{R}^{k}} \widehat{M}=\left\{z=\left(z^{(1)}, \ldots, z^{(k)}\right) \in \mathbb{R}^{k} \mid z \in \widehat{M}\right\} .
\end{aligned}
$$

По определению $k$ имеем, что $P_{M} 0 \subset \widehat{M}$ и, следовательно, $\operatorname{pr}_{\mathbb{R}^{k}}\left(P_{M} 0\right) \subset M^{\prime}$, т. е. оба множества из (13) непусты, причем $M^{\prime}$ содержится в конечномерном координатном подпространстве $\mathbb{R}^{k}$.

Пусть $\hat{z} \in \widehat{M}$. Понятно, что $\widehat{M} \cap \stackrel{\circ}{B}_{c_{0}}=\varnothing$, откуда $\left|\hat{z}^{(i)}\right|>1$ для некоторого $i$. Из (13) имеем, что $i \in\{1, \ldots, k\}$. Это влечет, что $\left|\operatorname{pr}_{\mathbb{R}^{k}} \hat{z}^{(i)}\right|>1$. Следовательно, $M^{\prime} \cap \stackrel{\circ}{B}_{\mathbb{R}_{k}}=\varnothing$.

Рассмотрим замыкание $\mathrm{cl} M^{\prime}$ множества $M^{\prime}$ в $\mathbb{R}^{k}$. Убедимся, что $P_{M^{\prime}} 0=$ $P_{\mathrm{cl} M^{\prime}}$ 0. Для этого рассмотрим последовательность $\left(y_{n}^{\prime}\right) \subset M^{\prime}, y_{n}^{\prime} \rightarrow y^{\prime} \in P_{\mathrm{cl} M^{\prime}} 0$. Выберем $\hat{y}_{n} \in \widehat{M}$ таким образом, что $\operatorname{pr}_{\mathbb{R}^{k}} \hat{y}_{n}=y_{n}^{\prime}$. Из (13) вытекает, что $\rho_{\mathbb{R}^{k}}\left(0, \mathrm{cl} M^{\prime}\right)=1$. Итак, $\left(\hat{y}_{n}\right)$ - минимизирующая последовательность из $\widehat{M} \subset M$ для 0 . Поскольку $M$ аппроксимативно компактно, то $\left(\hat{y}_{n}\right)$ содержит сходящуюся к некоторой точке $y \in M$ подпоследовательность. Ясно, что $\|y\|=1$, т. е. $y \in P_{M} 0$. Теперь из (13) имеем, что $y \in \widehat{M}$. Окончательно, $\operatorname{pr}_{\mathbb{R}^{k}} y=y^{\prime} \in M^{\prime}$, что и требовалось доказать. 
Норма пространства $c_{0}$ естественным образом индуцирует на $\mathbb{R}^{k} \ell^{\infty}$-норму, поэтому отождествим $\mathbb{R}^{k}$ с пространством $\ell^{\infty}(k)$; тогда т-связность замкнутого множества в $\ell^{\infty}(k)$ равносильна его $\ell^{1}$-вьпуклости в смысле $(2)$.

Множество $M$ по условию замкнуто и $\mathrm{m}$-связно. Установим, что $\mathrm{cl} M^{\prime} \subset \ell^{\infty}(k)$ будет $\ell^{1}$-выпуклым множеством. Пусть $u, v \in \operatorname{cl} M^{\prime},\left(u_{n}\right),\left(v_{n}\right) \in M^{\prime}, u_{n} \rightarrow u$, $v_{n} \rightarrow v$. Пусть $\hat{u}_{n}, \hat{v}_{n} \in \widehat{M}$ такие что, что $\operatorname{pr}_{\mathbb{R}^{k}} \hat{u}_{n}=u_{n}, \mathrm{pr}_{\mathbb{R}^{k}} \hat{v}_{n}=v_{n}$. По предложению 6 для каждого $n$ найдется монотонная дуга $\hat{\varkappa}_{n}(\cdot) \subset M$, соединяюшая $\hat{u}_{n}$ и $\hat{v}_{n}$. Из предложения 1 имеем, что $\widehat{\varkappa}_{n}(\cdot) \subset \widehat{M}$ и что $\varkappa_{n}(\cdot):=\operatorname{pr}_{\mathbb{R}^{k}} \widehat{\varkappa}(\cdot) \subset M^{\prime}$ является монотонной дугой, соединяюшей $u_{n}$ и $v_{n}$. В силу монотонности по $t$ координатных функций $\varkappa_{n}^{(i)}(\cdot), i=1, \ldots, k$, для каждого $n$ найдется точка $z_{n} \in \varkappa_{n}(\cdot)$ такая, что $\left|z_{n}-u_{n}\right|=\left|u_{n}-v_{n}\right| / 2$. Последовательность $\left(z_{n}\right) \subset \mathbb{R}^{k}$ обладает сходящейся подпоследовательностью. Пусть $z$ - ее предельная точка. Тогда $|z-u|=|u-v| / 2$ и $z \in \mathrm{m}(u, v)$ в силу (8).

Итак, множество $\operatorname{cl} M^{\prime} \subset \mathbb{R}^{k}$ замкнуто и $\ell^{1}$-вьпукло, причем $\stackrel{\circ}{B}_{\mathbb{R}^{k}}(0,1) \cap$ $\operatorname{cl} M^{\prime}=\varnothing, \rho_{\mathbb{R}^{k}}\left(0, M^{\prime}\right)=1$ и $P_{\mathrm{cl} M^{\prime}} 0=P_{M^{\prime}} 0$. Применяя (2), имеем, что $\mathrm{cl} M^{\prime}-$ солнце в $\ell^{\infty}(k)$. Пусть $y_{0}^{\prime}-$ солнечная точка из $\mathrm{cl} M^{\prime} \subset \ell^{\infty}(k)$ для 0 ; по доказанному вьше вьполняется $y_{0}^{\prime} \in M^{\prime}$. Пусть $y_{0} \in \widehat{M}$ - такая точка, что $\operatorname{pr}_{\mathbb{R}^{k}} y_{0}=y_{0}^{\prime}$.

Переставляя при необходимости координаты $1, \ldots, k$, без ограничения обшности считаем, что $y_{0}^{\prime}=\left(1, \ldots, 1, \eta^{(l+1)}, \ldots, \eta^{(k)}\right)$. Тогда

$$
y_{0}=\left(1, \ldots, 1, \eta^{(l+1)}, \ldots, \eta^{(k)}, \eta^{(k+1)}, \ldots\right), \quad\left|\eta^{(j)}\right|<1, \quad j \geqslant l+1 .
$$

Поскольку сl $M^{\prime}$ - солнце в $\ell^{\infty}(k)$, то по лемме А имеем

$$
\stackrel{\circ}{K}_{\mathbb{R}^{k}}\left(y_{0}^{\prime}, 0\right) \cap M=\varnothing ;
$$

здесь в силу (6) и (14)

$$
\begin{aligned}
\stackrel{\circ}{K}_{\mathbb{R}^{k}}\left(y_{0}^{\prime}, 0\right) & =\left\{z \in \mathbb{R}^{k} \mid z^{(i)}<1 \text { для всех } i=1, \ldots l\right\}, \\
K & \stackrel{\circ}{K}\left(y_{0}, 0\right)=\left\{z \in c_{0} \mid z^{(i)}<1 \text { для всех } i=1, \ldots l\right\} .
\end{aligned}
$$

Установим, что

$$
\stackrel{\circ}{K}\left(y_{0}, 0\right) \cap M=\varnothing .
$$

Из соотношений (15) и (13) вытекает, что $\widehat{M} \cap \stackrel{K}{K}\left(y_{0}, 0\right)=\varnothing$. Предположим, что (16) неверно. Тогда найдется точка $v \in M \cap K\left(y_{0}, 0\right), v \notin \widehat{M}$. Так как замкнутое множество $M$ по условию $\mathrm{m}$-связно, то по предложению 6 найдется монотонная дуга $\varkappa(\cdot):[0,1] \rightarrow M$, соединяюшая $v$ и $y_{0}$; считаем $\varkappa(0)=v, \varkappa(1)=y_{0}$.

Обозначим $\underline{J}=\left\{j \in[l+1, k]|| v^{(j)} \mid \geqslant 1\right\}, \bar{J}=\left\{j>k|| v^{(j)} \mid \geqslant 1\right\}$. Поскольку $v \in M \backslash \widehat{M}$, то $\bar{J} \neq \varnothing ;$ кроме того, $\bar{J}$ и $\underline{J}$ конечны. Положим

$$
t^{*}=\max \left\{t \in[0,1] \mid \text { найдется номер } j \in \bar{J} \cup \underline{J} \text { такой, что }\left|k^{(j)}(t)\right| \geqslant 1\right\} .
$$

Поскольку $y_{0} \in \widehat{M}$ и кривая $\varkappa(\cdot)$ непрерывна, то $t^{*}<1$.

Далее, пусть $\underline{J}^{*}=\left\{j \in \underline{J}|| \varkappa^{(j)}\left(t^{*}\right) \mid=1\right\}, \bar{J}^{*}=\left\{j \in \bar{J}|| \varkappa^{(j)}\left(t^{*}\right) \mid=1\right\}$. По крайней мере одно из этих множеств непусто. Предположим, что $\bar{J}^{*} \neq \varnothing$. Тогда $\left\|\varkappa\left(t^{*}\right)\right\|=1$. Имеем $\varkappa\left(t^{*}\right) \in P_{M} 0 \subset \widehat{M}$, что противоречит определению числа $k$.

Теперь считаем, что $\underline{J}^{*} \neq \varnothing\left(\bar{J}^{*}=\varnothing\right.$ по доказанному вьше). Имеем $v \in \widehat{M}$, но ранее доказано, что $v \notin \widehat{M}$. Противоречие.

Итак, предположение, что $K\left(y_{0}, 0\right) \cap M \neq \varnothing$, было неверно, откуда $M$ - солнце в $c_{0}$ по лемме А. Теорема доказана. 
ТЕОРемА 4. М-связное чебишевское множество в с является солнцем.

ДокАЗАТЕЛЬСтво. Пусть $M-\mathrm{m}$-связное чебьшевское множество в $c_{0}$. Без ограничения общности считаем $0 \notin M, \rho(0, M)=1$. Обозначим $y=P_{M} 0$. Переставляя при необходимости координаты, имеем, что $y^{(1)}=\cdots=y^{(k)}=1$, $\left|y^{(j)}\right|<1$ при $j>k$.

Покажем, что множество $M$ является солнцем, для этого воспользуемся леммой А. Предположим, что $z \in M \cap \stackrel{\circ}{K}(y, 0)$. По теореме 1 множество $M$ монотонно линейно связно, поэтому найдется монотонная дуга $k:[0,1] \rightarrow M$, соединяющая $y$ и $z, k(0)=y, k(z)=1$. Поскольку $y, z \in c_{0}$ и $\left|y^{(j)}\right|<1$ при $j>k$, из монотонности по $t$ координатных функций $k^{(j)}$ кривой $k(t)$ следует, что найдется $\varepsilon>0$ такое, что $\left|k^{(j)}(t)\right|<1$ при всех $j>k$ и $t \in(0, \varepsilon)$. Далее, поскольку $z \in \stackrel{K}{K}(y, 0)$, то в силу (6) имеем $z^{(i)}<1$ при $i=1, \ldots, k$. Поэтому найдется $\varepsilon^{\prime}>0$ такое, что $\left|k^{(i)}(t)\right| \leqslant 1$ при всех $i=1, \ldots, k$ и $t \in\left(0, \varepsilon^{\prime}\right)$.

Проверим, что $k^{(j)}(t)<1$ для всех $t \neq 0$. Действительно, если предположить, что $k^{\left(i_{0}\right)}\left(t_{0}\right)=1$ при некотором $i_{0}$ (понятно, что $1 \leqslant i_{0} \leqslant k$ и и $t_{0} \in(0,1)$, то из монотонности будем иметь $k^{\left(i_{0}\right)}(t)=1$ при всех $t \leqslant t_{0}$. Получаем, что при $t<\min \left\{\varepsilon, \varepsilon^{\prime}, t_{0}\right\}$ выполнено $\|k(t)\|=1$. Поскольку $k(t) \neq y$ при $t \neq 0$ и $k(t) \in M$ при всех $t$, то $k(t) \in P_{M} 0$ при $t<\min \left\{\varepsilon, \varepsilon^{\prime}, t_{0}\right\}-$ противоречие с тем, что $M$ является чебышевским множеством.

Итак, $k^{(i)}(t)<1$ для любого $t$, поэтому $k(t) \subset \stackrel{\circ}{B}(0,1)$ при всех достаточно мальх $t>0$. Однако поскольку $k(\cdot) \subset M$, то имеем противоречие с условием $\rho(0, M)=1$. Поэтому исходное предположение, что $M \cap \stackrel{\circ}{K}(y, 0)$ непусто, неверно и $M-$ солнце по лемме А. Теорема доказана.

Следующее утверждение непосредственно следует из теорем 1 и 3.

ТЕОрема 5. Пусть $M$ - солнще в $c_{0}$. Тогда $M$ монотонно линейно связно.

ЛЕмма 1. Пусть $X=c_{0}, c$ или $\ell^{\infty}$. Пусть $M \subset X u H_{m} \in \operatorname{cAff}_{m}(X)$, $m<\infty$. Предположим, что $x \in H_{m}, \quad x \notin M u M \cap H_{m} \neq \varnothing$. Пусть $y-$ солнечная точка из $M$ для $x$. Положим $y_{m}=\operatorname{pr}_{H_{m}} y$. Тогда

$$
\left\|x-y_{m}\right\|=\|x-y\|
$$

ДоКАЗАТЕЛЬСТВО. Без ограничения обшности считаем $x=0$ и $\|y\|=1$ и отождествим $H_{m}$ c $\mathbb{R}^{m}$. Рассмотрим случай $X=c_{0}$ и предположим, что (17) не выполнено. Тогда $\left|y^{(i)}\right|<1$ для всех $i=1, \ldots, m$ и найдутся номера $i_{1}, \ldots, i_{k}>m$ такие, что $\left|y^{\left(i_{j}\right)}\right|=1$ при $j=1, \ldots, k$ и $\left|y^{l}\right|<1$ при $l>m, j \neq i_{1}, \ldots, i_{k}$. В нашем случае опорный конус $K(y, 0)($ см. $(5),(6))$ имеет вид

$$
\stackrel{\circ}{K}(y, 0)=\left\{z \in c_{0} \mid z^{\left(i_{j}\right)} \operatorname{sign} y^{\left(i_{j}\right)}<1, j=1, \ldots, k\right\} .
$$

Поскольку $i_{1}, \ldots, i_{k}>m$, то $X_{m} \subset \stackrel{\circ}{K}(y, 0)$. Однако это невозможно, так как $M \cap H_{m} \neq \varnothing$, в то время как $\stackrel{\circ}{K}(y, 0) \cap M=\varnothing$ по лемме А.

Пусть теперь $X=c$. Предположим, что (17) неверно. Тогда $\left|y^{(i)}\right|<1$ при $i=1, \ldots, m$. Рассмотрим два случая:

a) $\left|y^{(j)}\right|<1$ для всех $j>m$;

b) $\left|y^{(j)}\right|=1$ при $j \in I_{1} \neq \varnothing$, $\min I_{1}>m,\left|y^{(j)}\right|<1$ при $j \in I_{2}, I_{1} \cup I_{2}=\mathbb{N}$. 
Напомним, что произвольньй линейньй непрерывный функционал на $c$ имеет вид

$$
f(x)=\alpha \lim x^{(j)}+\sum \beta_{j} x^{(j)}, \quad\|f\|=|\alpha|+\sum\left|\beta_{j}\right| .
$$

В случае а) $\mathcal{E}_{y}$ из $(6)$ состоит из единственного функционала $\hat{f}(x)=\lim x^{(j)}$. Тогда в силу (6) имеем

$$
\stackrel{\circ}{K}(y, 0)=\left\{z \in c \mid \lim z^{(j)}<1\right\} .
$$

В случае b) $\hat{f} \in \mathcal{E}_{y}$ тогда и только тогда, когда $\left|\lim y^{(j)}\right|=1$. Остальные элементы из $\mathcal{E}_{y}$ имеют вид $f^{j}(x)=x^{(j)} \operatorname{sign} y^{(j)}$ при $j \in I_{1}$. Имеем

$$
\begin{aligned}
& \stackrel{\circ}{K}(y, 0)=\bigcap_{j \in I_{1}}\left\{z \in c \mid f^{j}(z)<f^{j}(y)\right\} \\
& \stackrel{\circ}{K}(y, 0)=\left\{z \mid \lim z^{(j)}<1\right\} \cap\left(\bigcap_{j \in I_{1}}\left\{z \mid f^{j}(z)<f^{j}(y)\right\}\right),
\end{aligned}
$$

когда $\left|\lim y^{(j)}\right|<1$ и $\left|\lim y^{(j)}\right|=1$ соответственно.

Из (18) и (19) вытекает, что в случаях а) и b) опорный конус $\stackrel{\circ}{K}(y, 0)$ содержит подпространство $H_{m}$. Но это невозможно, поскольку $M \cap H_{m} \neq \varnothing$ и по лемме А $\stackrel{\circ}{K}(y, 0) \cap M=\varnothing$.

Пусть $X=\ell^{\infty}$. Рассмотрим произвольный ограниченный линейный функционал $f$ на $\ell^{\infty},\|f\|=1, f(y)=1$. Тогда $f(z)=\int z d \varphi$, где $\varphi$ - позитивная конечно аддитивная мера на $\mathbb{N},\|f\|=\operatorname{Var} \varphi$. Предположим, что $\left|y^{(j)}\right|<1$ для $j=1, \ldots, m$. Тогда, поскольку $\|f\|=1$ и $f(y)=1$, мы заключаем, что $\varphi(1, \ldots, m)=0$, т. е. $f\left(H_{m}\right)=0$. Это влечет, что опорньй конус $\stackrel{K}{K}(y, 0)$ содержит подпространство $H_{m}$. Это невозможно, так как $u, v \in M \cap H_{m}$, а с другой стороны, по лемме А $\stackrel{\circ}{K}(y, 0) \cap M=\varnothing$. Итак, предположение $\left|y^{(j)}\right|<1$ для $j=1, \ldots, m$ было неверно, поэтому (17), а вместе с ним и лемма 1 доказаны.

Tеорема 6. Пусть $M$ - солнце в $X=c_{0}$, с или $\ell^{\infty}$, и пусть $H_{n}$-n-мерное аффинное координатное подпространство в $X, H_{n} \cap M \neq \varnothing$. Тогда:

1) $M \cap H_{n}$ m-связно.

Это утверждение әквивалентно любому из следующих условий:

2) $M \cap H_{n}$ монотонно линейно связно;

3) $M \cap H_{n} k$-связно для всех $k \in \mathbb{Z}_{+}$;

4) $M \cap H_{n} P$-ациклично в $H_{n}$;

5) $M \cap H_{n} V$-ациклично в $H_{n}$;

6) $M \cap H_{n}-$ солнце в $H_{n}$.

Кроме того, $M \cap H_{n}$ монотонно линейно связно.

Ответ на вопрос, является ли пересечение $M \cap H$ солнщем в $H$, где $M$ - солнце в $X=c_{0}, c$ или $\ell^{\infty}$ и $H \subset X-$ координатное аффинное подпространство, $\operatorname{dim} H=$ $\operatorname{codim} H=\infty$, остается открытым. 
ДОКАЗАТЕЛЬСТВО ТЕОРЕМЫ 6 аналогично доказательству теоремы 3. Как и ранее, отождествим $H_{n} \mathrm{c} \ell^{\infty}(n)$.

Предположим, что условие 1) доказано. Тогда монотонная линейная связность множества $M \cap H_{n}$ следует из леммы В. Утверждение 1$\left.) \Rightarrow 4\right)$, т. е. $P$-ацикличность солнц в $\ell^{\infty}(n)$, доказано Брауном в теореме А. Для компактного множества в конечномерном пространстве его $V$-ацикличность равносильна его $P$-ацикличности $[24$, следствие 1.6 .2$]$, поэтому имеем 4$) \Leftrightarrow 5)$. В [2, теорема 2] доказано, что $3) \Leftrightarrow 4)$. Ограниченно компактное $P$-ацикличное множество в банаховом пространстве является солнцем. Из этого результата Власова [11, теорема 4.4] вытекает 5$) \Rightarrow 6$ ). Поскольку $H_{n}$ можно отождествить с $\ell^{\infty}(n)$, а последнее является $(B M)$-пространством, то по теореме А имеем 6$) \Rightarrow 1$ ). Эквивалентность 1) $\Leftrightarrow 2$ ) установлена в (2).

Докажем утверждение 1). Предположим противное: найдется солнще $M \subset X$, не являющееся m-связным в некотором конечномерном координатном аффинном подпространстве $H_{n}$ в $X$. Не ограничивая общности, отождествим $H_{n}$ с $\left(\mathbb{R}^{n},\|\cdot\|_{\infty}\right)$, где $\|\cdot\|_{\infty}$ - обычная максимум-норма. Тогда по определению существуют точки $u, v \in M \cap H_{n}$ такие, что $(\mathrm{m}(u, v) \backslash\{u, v\}) \cap M=\varnothing$. Рассмотрим $\ell^{1}$-норму $|\cdot|_{1}$ на $H_{n}$ как ассоциированную (в смысле (8), (9)).

Пусть $H_{m}$ - минимальное координатное аффинное подпространство, которое содержит $u$ и $v$ (к примеру, если $u^{(i)} \neq v^{(i)}$ для всех $i=1, \ldots, n$, то $H_{m}=$ $\left.H_{n}\right)$. Без ограничения общности отождествим $H_{m} \mathrm{c}\left\{x=\left(x^{(1)}, \ldots, x^{(m)}, 0, \ldots\right)\right\}$. Теперь доказательство повторяет рассуждения, проведенные при доказательстве теоремы 2. Предположим, что $x_{0} \in A_{\varepsilon}, x_{0} \in H_{n},\left|x_{0}-u\right| \geqslant(1 / 2)|u-v|$ и что $y$ - солнечная точка для $x_{0}$ (ясно, в общем случае $y \notin H_{m}$ ). Для простоты обозначений положим $x_{0}=0$.

Определим точку $y_{m}$ как координатную проекцию точки $y$ на $H_{m}: y_{m}=\left(y^{(1)}, \ldots\right.$ $\left.\ldots, y^{(m)}, 0, \ldots\right)$. По лемме 1 имеем

$$
\left\|y_{m}\right\|=\|y\| .
$$

Поскольку $y$ - солнечная точка для 0 и $u \in M$, то $[y, u] \cap \stackrel{\circ}{B}(0,\|y\|)=\varnothing$ (см., например, $[11$, лемма 3.1$])$. Из этого следует, что $\left[y_{m}, u\right] \cap B(0,\|y\|)=0$. В действительности, из того, что $u \in H_{m}$, мы, используя (20), получаем

$$
\sup \left|\alpha u^{(i)}-(1-\alpha) y^{(i)}\right| \geqslant \sup \left|\alpha u^{(i)}-(1-\alpha) y_{m}^{(i)}\right| \geqslant 1
$$

для всех $0<\alpha<1$. Отсюда имеем

$$
\stackrel{\circ}{B}(0,\|y\|) \cap\left[y_{m}, y_{m}-\left(y_{m}-u\right)\right]=\varnothing .
$$

Вследствие (4) получаем

$$
B(0,\|y\|) \cap\left(\mathrm{m}\left(y_{m}, y_{m}-u\right) \backslash\left\{y_{m}\right\}\right) \neq \varnothing,
$$

что эквивалентно $B\left(y_{m},\|y\|\right) \cap(\mathrm{m}(0, u) \backslash\{0, u\}) \neq \varnothing$. Как и раньше, пусть $w \in$ $B\left(y_{m},\|y\|\right) \cap(\mathrm{m}(0, u) \backslash\{0, u\})$. Тогда $w \in H_{m}$. Так как $0 \in \mathcal{O}_{\varepsilon}(M)$ и $y \in M$, то $\|y\|<\varepsilon$, откуда $\left\|y_{m}-w\right\|<\varepsilon,\|y-w\|<\varepsilon$, т. е. $w \in A_{\varepsilon} \cap X_{m}$. Далее рассуждаем, как при доказательстве утверждения 3 ) теоремы 3 . Теорема доказана.

Работа была начата во время пребывания автора в университете г. Лилль (Франция). Автор благодарен профессору Э. Кефелеку за гостеприимство и научное сотрудничество. 


\section{Список литературы}

1. Brown A.L. Suns in normed linear spaces which are finite-dimensional // Math. Ann. 1987. V. 279. P. $87-101$.

2. Brown A. L. On the connectedness properties of suns in finite dimensional spaces // Proc. Cent. Math. Anal. Aust. Natl. Univ. 1988. V. 20. P. 1-15.

3. Brown A. L. Suns in polyhedral spaces // Seminar of Math. Analysis. Proceedings. Univ. Malaga and Seville (Spain), Sept. 2002 - Feb. 2003 / Eds. D. G. Álvarez, G. Lopez Acedo, R. V. Caro. Sevilla: Universidad de Sevilla, 2003. P. 139-146.

4. Braess D. Geometrical characterizations for nonlinear uniform approximation // J. Approx. Theory. 1974. V. 11. P. 260-274.

5. Berens H., Hetzelt L. Die Metrische Struktur der Sonnen in $\ell^{\infty}(n) / /$ Aequat. Math. 1984. V. 27. P. 274-287.

6. Алимов А.P. Геометрическая характеризация строгих солнц в $\ell^{\infty}(n) / /$ Матем. заметки. 2001. Т. 70. № 1. С. 3-11.

7. Franchetti $C$., Roversi $S$. Suns, $M$-connected sets and $P$-acyclic sets in Banch spaces. Preprint № 50139 / Instituto di Matematica Applicata "G. Sansone", 1988. P. 1-29.

8. Алимов А.P. Геометрическое строение чебышевских множеств в $\ell^{\infty}(n) / /$ Функцион. анализ и его прилож. 2005. Т. 38. № 1. С. 1-10.

9. Alimov A.R. Characterisations of Chebyshev Sets in $c_{0}$ // J. Approx. Theory. 2004. V. 129. № 2. P. 217-229.

10. Стечкин С. Б. Избранные труды. Математика. М.: Наука, 1998.

11. Власов Л. П. Аппроксимативные свойства множеств в линейных нормированных пространствах // УМН. 1973. Т. 28. №6. С. 3-66.

12. Балаганский В. С., Власов Л. П. Проблема выпуклости чебышевских множеств // УМН. 1996. T. 51. №6(312). С. 125-188.

13. Карлов М.И., Царьков И. Г. Выпуклость и связность чебышевских множеств и солнц // Фундам. и прикл. матем. 1997. Т. 3. № 4. С. 967-978.

14. Власов Л. П. Понятие аппроксимативной компактности и его варианты // Матем. заметки. 1974. Т. 16. № 2. С. 337-348.

15. Kenderov P.S., Giles J.R. On the structure of Banach spaces with Mazur's intersection property // Math. Ann. 1991. V. 291. № 3. P. 463-474.

16. Whitfield J. H. M., Zizler V. Mazur's intersection property of balls for compact convex sets // Bull. Aust. Math. Soc. 1987. V. 35. P. 267-274.

17. Köthe G. Topological vector spaces. I. Berlin: Springer-Verlag, 1969.

18. Menger K. Untersuchungen über allgemeine Metrik // Math. Ann. 1928. V. 100. P. 75-163.

19. Gordeo-Erasquin D., McCann R. J., Schmuckenschläger M. A Riemannian interpolation inequality à la Borell, Brascamp and Lieb // Invent. Math. 2001. V. 146. P. 219-257.

20. Власов Л. П. Чебышевские множества и некоторые их обобщения // Матем. заметки. 1968. T. 3. № 1. C. 36-41.

21. Кощеев $B . A$. Связность и аппроксимативные свойства множеств в линейных нормированных пространствах // Матем. заметки. 1975. Т. 17. № 2. С. 193-204.

22. Кощеев B.A. Связность и солнечные свойства множеств в линейных нормированных пространствах // Матем. заметки. 1976. Т. 19. № 2. С. 267-278.

23. Кощеев B. A. Пример несвязного солнца в банаховом пространстве // Матем. заметки. 1979. T. 26. № 1. C. 89-92.

24. Brown A.L. Chebyshev sets and the shapes of convex bodies // Methods of Functional Analysis in Approximation Theory / Ed. C.A. Micchelli. Internat. Series of Numerical Math. V. 76. Basel: Birkhäuser-Verlag, 1986. P. 97-121. 